\title{
Atopisches Ekzem kurz und gut
}

A usführlich wird im vorgestellten Buch das Wissen über Epidemiologie, Ätiologie, Pathogenese, Klinik und Differenzialdiagnostik des atopischen Ekzems dargestellt. Ein umfangreiches Literaturverzeichnis mit Hinweisen auf neuere Untersuchungen unterstreicht den enormen Erkenntniszuwachs, der auf diesem Gebiet in den letzten Jahren erzielt worden ist. Klinische Sonderformen, besondere Manifestationen bzw. Lokalisationen werden beschrieben und auf zahlreichen Farbabbildungen dargestellt. Weiter werden eingehend deren diagnostische, differenzialdiagnostische und auch therapeutische Besonderheiten beleuchtet. Auch wird auf assoziierte Erkrankungen des atopischen Ekzems eingegangen, so wird beispielsweise auf die nur allzu oft vergessene Augenbeteiligung hingewiesen oder es werden verschiedene Ursachen einer Wachstumsretardierung im Kindesalter besprochen.

Ein besonderer Schwerpunkt liegt auf Diagnostik und Therapie des atopischen Ekzems. Auch hier schlägt sich das zunehmend Verbreitung findende Konzept einer exogenen Auslösung des atopischen Ekzems nieder. Viele Triggerfaktoren werden benannt, die in der Diagnostik abgeklärt werden müssen. Altbekannte Behandlungsmethoden und neuere therapeutische Ansätze werden ausführlich dargestellt. Auch komplementäre Therapieverfahren werden kritisch beleuchtet. Wer schon immer der Bioresonanztherapie oder Elektroakupunktur nach Voll verständnislos gegenüber stand, erhält hier eine „Erklärung" dieser Verfahren und kann auch die Quellenangaben der Studien finden, welche die Ineffektivität dieser Methoden gezeigt haben.

Wer sich kurz und knapp - aber dennoch fundiert - informieren will, ist mit diesem Buch bestens beraten.

Dr. Franziska Ruëff, München

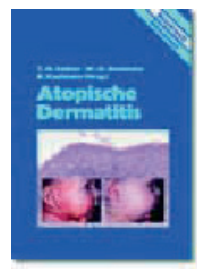

Zollner TM, Boehncke W-H, Kaufmann R. Atopische Dermatitis. Berlin, Wien: Blackwell Wissenschaftsverlag, 2002. 64,95€. ISBN 3-89412-494-6

\section{Immunologie für Bequeme?}

$\mathrm{D}$ er Liegestuhl auf dem Umschlag scheint eine entspannte Lektüre zu verheißen: „Immunologie auf 70 Seiten" präsentiert jedoch dichtgedrängt die grundlegenden Fakten der Immunologie. Die Entwicklung der verschiedenen immunologisch wichtigen Zellen aus einer Stammzelle wird dargestellt, die Strukturen und Eigenschaften der verschiedenen Antikörper und die immunologische Labordiagnostik werden erläutert, ein kurzer Abriss der Immungenetik wie auch ein Überblick über die Immunpathologie werden gegeben.

„Immunologie auf 70 Seiten“ ist ein kurz gefasstes Lernbuch, kein Lehrbuch, und erhebt damit auch keinen Anspruch auf Vollständigkeit. Mittels eines klar gegliederten, sach- lich strukturierten Kurztextes und übersichtlichen Schaubildern ist es ideal zum Lernen und Wiederholen des Prüfungsstoffs während des Medizinstudiums, aber auch im späteren Verlauf der Medizinerkarriere empfiehlt es sich für eine Rekapitulation oder schnelles Nachschlagen von einmal Gelerntem.

Dr. Inga Hundorf, Heidelberg

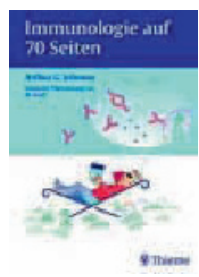

Johnson AG (deutsch von Kraft M). Immunologie auf 70 Seiten. Stuttgart, Heidelberg, New York: Georg-Thieme-Verlag, 2001. 9,95 €. ISBN 3-13126-471-3 